\title{
PROMOTION AND CONTROL OF TURBULENT MIXING OF HOT AND COLD AIRFLOWS IN T-JUNCTION
}

\author{
${ }^{* 1}$ Takuya Matsuda, ${ }^{* 1}$ Masafumi Hirota, ${ }^{* 2}$ Hideo Asano, ${ }^{* 3}$ Shuichiro Hori, \\ ${ }^{* 1}$ Naoki Maruyama and ${ }^{* 1}$ Akira Nishimura \\ ${ }^{* 1}$ Department of Mechanical Engineering, Mie University, 1577 Kurimamachiya-cho, Tsu-city, 514-8507, Japan \\ 412m147@m.mie-u.ac.jp, hirota@mach.mie-u.ac.jp, maruyama.naoki@mie-u.ac.jp,nisimura@mach.mie-u.ac.jp \\ *2 Thermal Systems Performance Development Div., DENSO CORPORATION, 1-1 Showa-cho, Kariya 448-8661, Japan \\ ${ }^{* 3}$ Akashi Works, Kawasaki Heavy Industry, 1-1, Kawasaki-cho, Akashi, Hyogo 673-8666, Japan
}

\begin{abstract}
An experimental study was made on the promotion and control of turbulent mixing of hot and cold airflows in a T-junction with rectangular cross sections. A delta-wing row was attached on the bottom wall of the main channel before the flow merging to promote and control the turbulent thermal mixing. The mean temperature and velocity distributions were measured in several cross sections after the flow merging by thermocouples and PIV, respectively. The development of the thermal mixing layer could be promoted effectively by the delta wings and the thermal mixing could be controlled by changing the angle of attack of wings. Longitudinal vortices produced by the delta wings disappeared just after the merging of two flows. High turbulence generated by the interaction of those vortices and branch flow was, however, maintained to further downstream cross sections and contributed to the promotion of the thermal mixing.
\end{abstract}

\section{INTRODUCTION}

A cross-flow type T-junction in which two flows with different velocities, temperatures, and/or concentrations are mixed is used in various thermal hydraulic equipments such as combustion chamber and piping system in power plants [1]-[3]. The mixing T-junctions is also found in the HVAC (Heating, Ventilating, Air-Conditioning) unit used for an automobile air-conditioning system. A conceptual diagram of the automobile HVAC unit is presented in Fig. 1, which contains a fan, an evaporator and a heater-core in a plastic housing [4][5]. All air taken by the fan is once cooled by the evaporator to reduce humidity, and a part of this cold air is heated by the heater-core. The temperature of air blown into the cabin is controlled by mixing them at appropriate flow-rate ratios, which are determined by the opening of the air-mix door that is located between two heat exchangers. In the mixing zone of the HVAC unit, hot and cold airflows impinge at nearly right angles as shown in Fig. 1, and this flow configuration can be modeled by the turbulent thermal mixing of hot and cold airflows in a cross-flow type $\mathrm{T}$-junction with rectangular cross sections.

Nowadays, the downsizing the HVAC unit is an important issue in the automobile A/C system, and it is also required that the temperature distribution in the cabin be

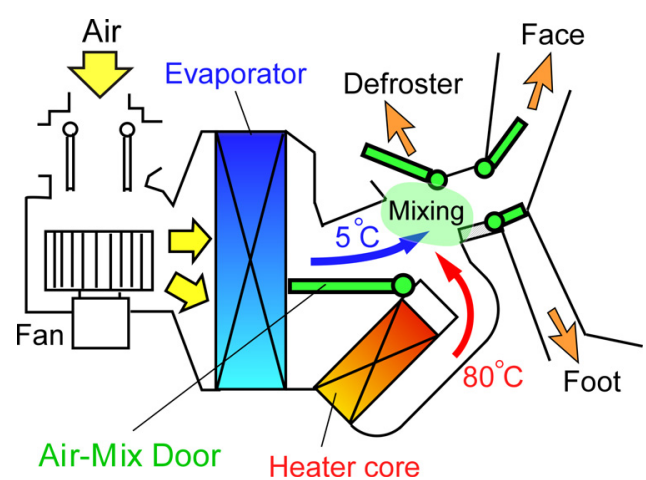

Fig. 1 Structure of HVAC unit for automobile A/C

controlled locally depending on the operation mode of the $\mathrm{A} / \mathrm{C}$ system. Therefore, the promotion and control of turbulent mixing of hot and cold airflows are key subjects to solve these problems in the HVAC development. Active methods that need external power inputs, such as synthetic jets and micro actuators, are quite effective to the mixing promotion/control of two flows [6][7], but the passive methods without any external power inputs are required in the practical application to the HVAC unit.

With these points as background, we made an experimental study on the promotion and control of turbulent mixing of hot and cold airflows in the T-junction with rectangular cross sections. We adopted delta wings [8][9] as a promoter of the turbulent mixing of two flows, and tried to control the degree of mixing by changing the angle of attack of the wings. In this paper, we at first show the characteristics of the velocity and temperature fields in a simple T-junction without a mixing promoter/controller, and explain the problems in the promotion and control of the thermal mixing. Then, based on the results of the velocity and temperature measurements and flow visualization, we demonstrate the effectiveness of the delta wings as a mixing promoter/controller. Finally, the mechanism of mixing promotion and control by the delta wings is discussed.

\section{EXPERIMENTAL APPARATUS}

Figure 2 shows a schematic diagram of the standard test channel that has no mixing promoters. The vertical branch channel was joined to the horizontal main channel at right angles to form a T-junction. The specification of this 


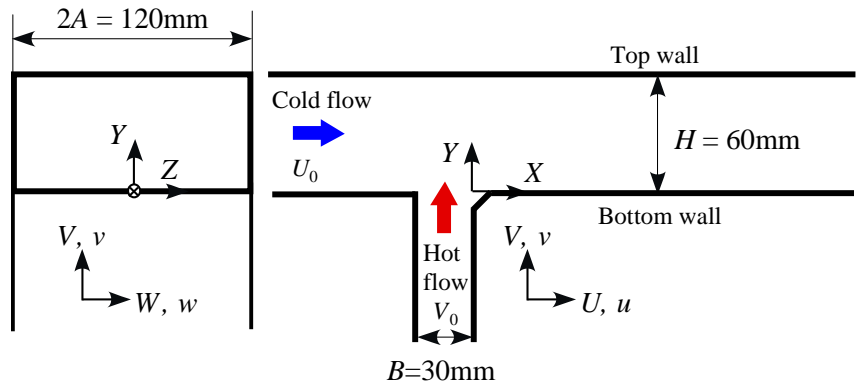

Fig. 2 Standard channel without mixing promoter

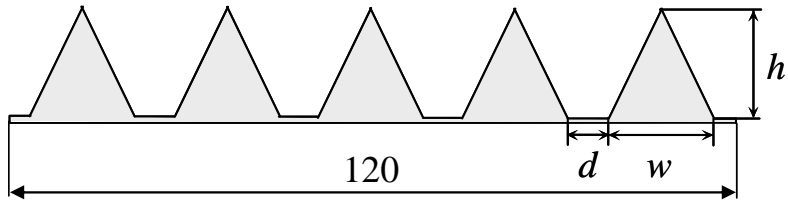

Fig. 3 Example of tested delta-wing row $(n=5, d / w=0.33)$

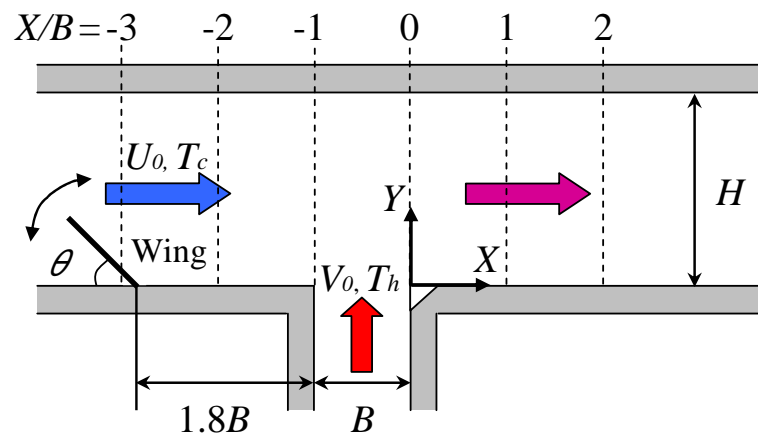

Fig. 4 Test channel with the delta-wing row

channel was essentially the same as that used in the preceding study [5] except that the second edge (edge in the downstream side) of the T-junction was planed off. This is because the flow separation that occurs at this edge increases the pressure loss but high turbulence generated around the separation bubble does not contribute to the turbulent thermal mixing of two flows [7]. The scale of the separation bubble was reduced by chamfering this edge. Airflows in the main and branch channels were merged in the T-junction after flowing through heat exchangers, settling chambers and contraction flow nozzles. The height of the main channel $H$ was $60 \mathrm{~mm}$ and the width $2 A$ was 120 $\mathrm{mm}$. The width of the branch was the same as that of the main channel $(120 \mathrm{~mm})$, and the $X$-way length of the branch cross section $B$ was $30 \mathrm{~mm}$. The chamfered length of the second edge was $6 \mathrm{~mm}=0.2 B$. The coordinate system is also shown in Fig. 2. In a real HVAC unit, the exit of the mixing zone corresponds to the cross section of $X / B=2-3$. The mean and fluctuating velocity components are denoted as $U, V, W$, and $u, v, w$, respectively.

Figure 3 shows an example of delta wings tested in this study. We tested several kinds of delta-wing rows with different wing number $n$, wing height $h$ and wing clearance $d$. In this paper, however, the results obtained with the wing row with $n=5, h / H=0.45$ and $d / w=0.33$ are presented, which was most effective as a mixing promoter. The vertical angle and the aspect ratio [8] of each delta wing was $37^{\circ}$ and 1.33 , respectively As shown in Fig. 4, it was attached to the bottom wall of the main channel before the T-junction. We expected that turbulence could be enhanced inside the thermal mixing layer by the interaction between longitudinal vortices generated by wings in the main flow and the branch flow that merged with it in the T-junction. This high turbulence might act effectively on the vertical development of the thermal mixing layer. Moreover, it was expected that the turbulence intensity and resulting turbulent mixing could be controlled by changing the angle of attack of wings. The installation position of the delta-wing row was optimized based on the mean temperature distributions measured in preliminary experiments, and it was determined as $X / B=-2.8$ on the bottom wall of the main channel. The angle of attack of wings $\theta$ was varied from $10^{\circ}$ to $70^{\circ}$ at $10^{\circ}$ pitches.

Experiments were conducted keeping the main-flow Reynolds number at $2.5 \times 10^{4}$ which was defined based on the hydraulic diameter of the main channel $D_{h}$ and the bulk velocity in it before the mixing $U_{0}$. The bulk velocity in the branch $V_{0}$ was determined so that the velocity ratio $V R=$ $V_{0} / U_{0}$ be changed from 0.5 to 1.0 . Velocity distributions were measured by 2-D PIV under an isothermal condition. For statistical analyses, the mean velocity and turbulence intensities were computed by an ensemble average of 500 instantaneous velocity fields. In the measurement of the mean temperature distributions, the bulk temperature of the branch flow was set at $60^{\circ} \mathrm{C}\left(=T_{h}\right)$ while the main flow was in room temperature $\left(=T_{c}\right)$. The time-averaged local temperature distributions were measured by a thermocouple rake. By a close comparison of velocity and temperature distributions, we examine the mechanism of turbulent mixing promotion and control of two flows by the delta-wing row.

\section{RESULTS AND DISCUSSION}

\subsection{Temperature and velocity fields in the standard channel}

In this section, the characteristics of the velocity and mean temperature fields in the standard channel without mixing promoters are overviewed, and based on them the strategy for promoting the thermal mixing is explained.

Distributions of the non-dimensional mean temperature $\left(T-T_{c}\right) /\left(T_{h}-T_{c}\right)$ in the channel measured in half cross sections of $Z / A>0$ at $X / B=0,1,2$ and 3 with $V R=0.5$ are shown in Fig. 5. The mean temperature shows quite uniform distributions in the spanwise direction (Z-direction), and the development of the thermal mixing layer is quite slow. In particular, in the cross sections of $X / B>2$ corresponding to the exit of the mixing zone of the HVAC unit, the upward development of the thermal mixing layer is relatively limited in comparison with the downward development.

This limited development of the thermal mixing layer in the upward direction is closely related to the distribution of turbulence intensity $v^{\prime} / U_{0}$, where $v^{\prime}$ is RMS of the fluctuating velocity component in the vertical ( $Y$-axis) direction $v$. 


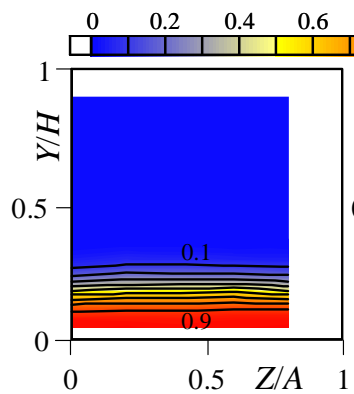

(a) $X / B=0$

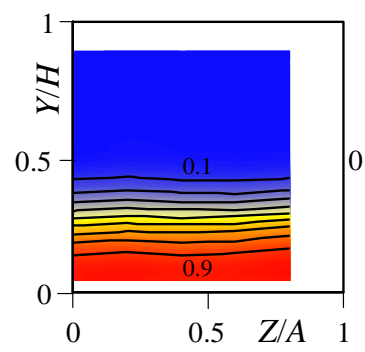

(c) $X / B=2$

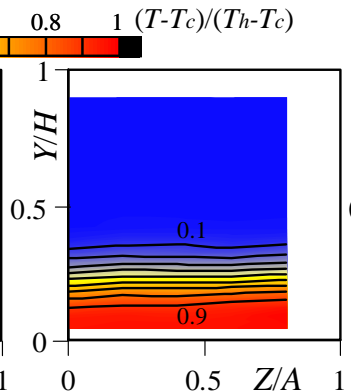

(b) $X / B=1$

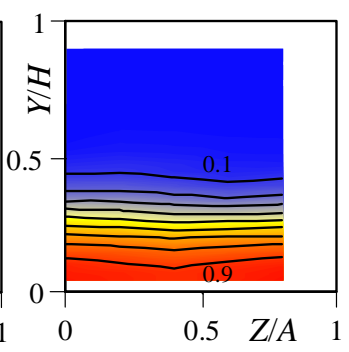

(d) $X / B=3$

Fig. 5 Mean temperature distributions in standard channel

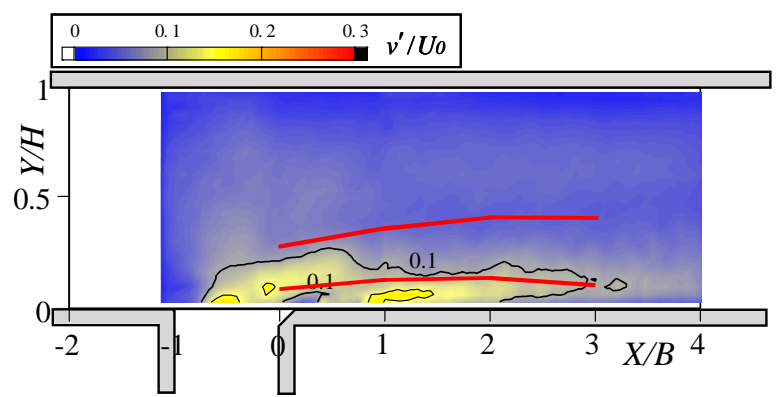

Fig. 6 Distribution of turbulence intensity $v^{\prime} / U_{0}$ in $Z / A=0$

Figure 6 shows the distributions of $v^{\prime} / U_{0}$ measured in the symmetric plane of the channel. The red lines in the figure show the locations at which $\left(T-T_{c}\right) /\left(T_{h}-T_{c}\right)=0.1$ (upper boundary of the thermal mixing layer) and $\left(T-T_{c}\right) /\left(T_{h}-T_{c}\right)=$ 0.9. $v^{\prime}$ near the upper boundary is quite smaller than that near the lower boundary. The heat transfer between the hot and cold airflows is dominated by the turbulent heat flux $\overline{v t}$ and its main production term is expressed as $\overrightarrow{v^{2}} \partial T / \partial Y$. Therefore, the production of $\overline{v t}$ is quite small in the upper part of the thermal mixing layer, and this causes such a limited development of the thermal mixing layer in the upward direction as observed in Fig. 5.

From these results in the standard channel, it is expected that the development of the thermal mixing layer can be promoted effectively if $v^{\prime}$ is increased in its upper part. Among many options, we adopted a delta-wing row as a mixing promoter/controller considering feasibility to a real HVAC unit and potential to promote and control the mixing.

\subsection{Mean temperature distributions in the channel with the delta-wing row}

The distributions of mean temperature $\left(T-T_{c}\right) /\left(T_{h}-T_{c}\right)$ measured in a half cross section $(Z / A>0)$ at $X / B=2$ with a delta-wing row of $n=5, h / H=0.45$ and $d / W=0.33$ are

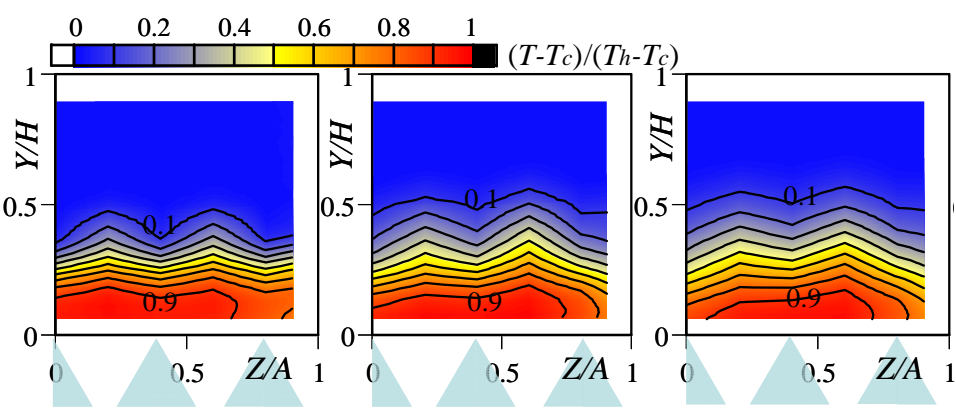

(a) $\theta=10^{\circ}$

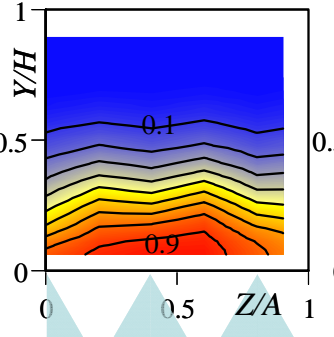

(b) $\theta=30^{\circ}$

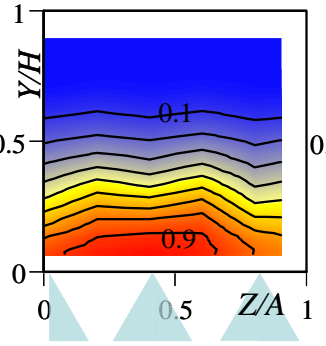

(c) $\theta=40^{\circ}$

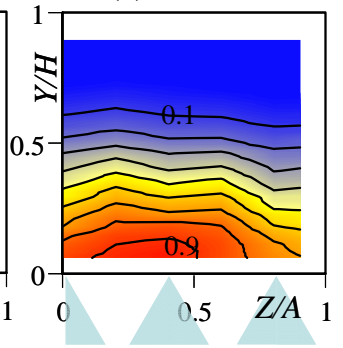

(f) $\theta=70^{\circ}$

(e) $\theta=60^{\circ}$

(d) $\theta=50^{\circ}$

Fig. 7 Variation of $\left(T-T_{c}\right) /\left(T_{h}-T_{c}\right)$ with $\theta$ at $X / B=2$

shown in Fig. 7. The streamwise location of this cross section corresponds to the exit of the mixing zone of a real HVAC unit. The angle of attack of the wings $\theta$ is changed from $10^{\circ}$ to $70^{\circ}$, and the triangles shown at the bottom of each figure correspond to the locations of the wings.

At $\theta=10^{\circ}$, the mean temperature shows an uneven distribution in the spanwise direction, and the thermal mixing layer near the trough of the wavy isotherms is thinner than that in the standard channel. This suggests that longitudinal vortices generated by wings influence the temperature distribution in the mixing layer. As $\theta$ is increased, the thermal mixing layer becomes thicker and the spanwise unevenness of the temperature distribution is gradually relieved. The development of the thermal mixing layer was saturated in $\theta>70^{\circ}$. These results demonstrate that the delta-wing row works well as a mixing promoter and the degree of mixing of two flows can be controlled by changing the angle of attack of the wings as expected. In a real HVAC unit, the spanwise uniformity of the mean temperature distribution is required to secure the comfortability of the cabin. As described above, the spanwise uniformity of the mean temperature distribution is improved as $\theta$ is increased. This mixing performance of the delta-wing row is preferred to the HVAC unit.

\subsection{Evaluation of mixing promotion and control}

In order to evaluate quantitatively the performance of the delta-wing row as a mixing promoter and controller, we defined the "mixedness." Figure 8 illustrates the concept of the mixedness. The blue lines in the figure show the spanwise-averaged non-dimensional mean temperature distribution measured in this study, and it is denoted as $T_{m}{ }^{*}$. The thickness of the thermal mixing layer $W$ is defined as the difference of the $Y$-coordinates at which $T_{m}{ }^{*}=0.1(Y=$ $\left.Y_{0.1}\right)$ and $T_{m}{ }^{*}=0.9\left(Y=Y_{0.9}\right)$, i.e., $W=Y_{0.1}-Y_{0.9}$. The violet 


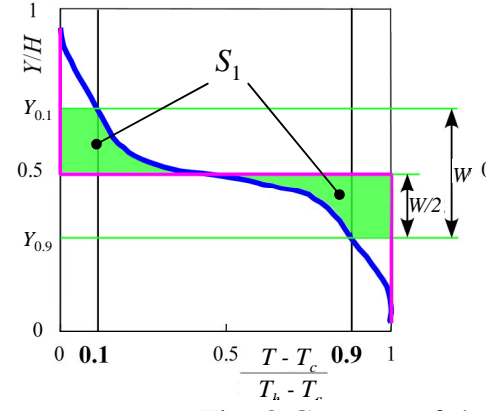

Fig. 8 Concept of the “mixedness” $M$

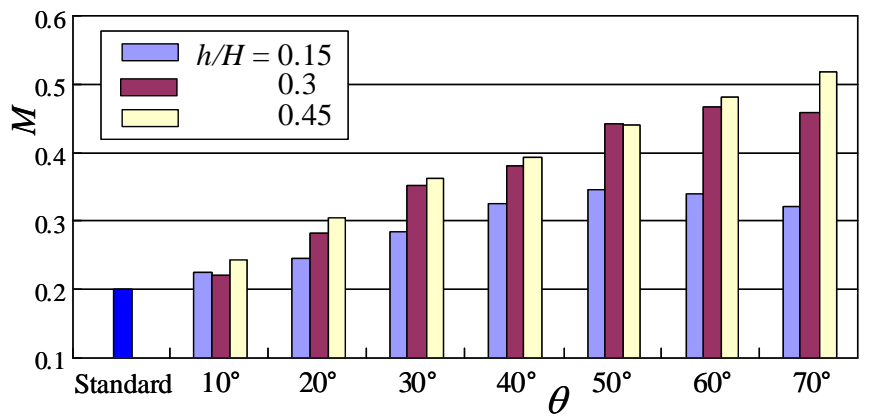

Fig. 9 Variation of the mixedness for $\theta$ with different height

line is the virtual dimensionless mean temperature distribution showing the condition that the hot and cold airflows are not mixed at all. In this case, the mean temperature is assumed to change from $T_{c}$ to $T_{h}$ like a step function at the center of the thermal mixing layer. This discontinuous mean temperature distribution is denoted as $T_{u m}{ }^{*}$ and expressed as follows.

$$
T_{u m} *= \begin{cases}0 & \left(Y>Y_{0.1}-W / 2\right) \\ 1 & \left(Y<Y_{0.1}-W / 2\right)\end{cases}
$$

The red line shows the mean temperature distribution ideal for the HVAC unit, in which $T_{m}{ }^{*}$ changes from $T_{m}{ }^{*}=$ 0.1 at $Y=Y_{0.1}$ to $T_{m}{ }^{*}=0.9$ at $Y=Y_{0.9}$ linearly, and this mean temperature distribution $T_{i d}{ }^{*}$ is expressed by the following equation.

$$
T_{i d} *=-\frac{0.8}{W}\left(Y-Y_{0.9}\right)+0.9
$$

Here, the area enclosed by $T_{m}{ }^{*}$ and $T_{u m}{ }^{*}$ is denoted as $S_{1}$, and that enclosed by $T_{i d}{ }^{*}$ and $T_{u m}{ }^{*}$ is denoted as $S_{2}$ (green areas in Fig. 8). That is,

$$
S_{1}=\int_{Y_{0.9}}^{Y_{0.1}}\left|T_{u m}{ }^{*}-T_{m}^{*}\right| d Y, \quad S_{2}=\int_{Y_{0.9}}^{Y_{0.1}}\left|T_{u m}{ }^{*}-T_{i d}^{*}\right| d Y
$$

From these parameters, the mixedness $M$ is defined by the following equation.

$$
M=\frac{S_{1}}{S_{2}} \times \frac{W}{H}
$$

$S_{1} / S_{2}$ means the deviation of the measured temperature distribution from ideal one, and $W / H$ is the dimensionless thickness of the thermal mixing layer. Thus, this mixedness can evaluate quantitatively the performance of the delta-wing row from viewpoints of not only its ability to promote and control the mixing of two flows but also the appropriateness of the mean temperature distribution of the mixed flow for the HVAC unit.

Figure 9 shows the variations of this mixedness $M$ to the angle of attack of the wings $\theta$. The results obtained for three delta-wing rows with different height $(h / H=0.15,0.3$ and 0.45 ) are compared with that for the standard channel. For all wing heights, the mixedness increases linearly as $\theta$ is increased. This result demonstrates again that the mixing of hot and cold flows can be controlled by changing the angle of attack of the delta wings. At the same $\theta$, larger mixedness can be obtained with higher wings. The maximum $M$ is attained with the wing row of $h / H=0.45$ at $\theta$ $=70^{\circ}$. We found that the mean temperature showed considerable non-uniform distributions in the spanwise direction with a higher wing row of $h / H=0.6$. From these results, it follows that the delta-wing row with the height of $h / H=0.45$ has the highest performance as a mixing promoter/controller among the wings tested in this study.

\subsection{Mean velocity and turbulence intensity in the channel with the delta-wing row}

At first, we show the streamwise evolution of secondary flow mean velocity vectors measured at $\theta=30^{\circ}$ in Fig. 10 . In a half cross section at $X / B=-2,0.8 B$ downstream the wing-row base, a pair of longitudinal vortices with a common-flow down [9][10] along the wing bisector is generated just downstream each wing. At $X / B=-1$ corresponding to the entrance of the flow-merging region, the downward flow is considerably weakened but a secondary flow pattern similar to that appeared at $X / B=-2$ is still observed. At $X / B=-0.5$, a clear vortex pattern is not observed and high and low upward velocity regions are distributed alternately in the spanwise direction. The low velocity regions correspond to the common-flow down regions observed at $X / B=-2$ and -1 . This suggests that a vortex structure is broken in the flow-merging region by the overwhelming upward flow from the branch, but the influence of the longitudinal vortices is still maintained in this region. Such footprints of the longitudinal vortices, however, disappear in the cross sections of $X / B>0$.

The results for a larger angle of attack $\theta=60^{\circ}$ are shown in Fig. 11. The vortex pattern is qualitatively similar to that observed at $\theta=30^{\circ}$, but the scale and intensity of vortices at $X / B=-2$ are larger than those for $\theta=$ $30^{\circ}$ and the vortices are clearly observed at $X / B=-1$ as well. The influence of the longitudinal vortices on the velocity distributions, i.e., high and low upward velocity regions, is still observed at $X / B=0$, then disappear in $X / B>1$.

Next, the distributions of turbulence intensity $v^{\prime} / U_{0}$ for $\theta$ $=30^{\circ}$ are shown in Fig. 12. This fluctuating velocity component contributes to the turbulent transport of heat in the $Y$-direction, i.e., the upward development of the thermal mixing layer. At $X / B=-2, v^{\prime}$ shows the local maximums in the regions between the wings. In the cross sections located further downstream, these large $v^{\prime}$ regions spread in both the spanwise and vertical directions and $v^{\prime}$ once decreases at $X / B=-1$ but increases to attain the maximum at $X / B=-0.5$. This large $v^{\prime}$ is generated by the large velocity 


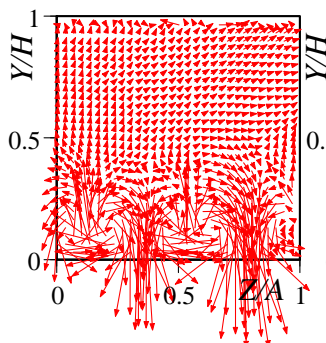

(a) $X / B=-2$

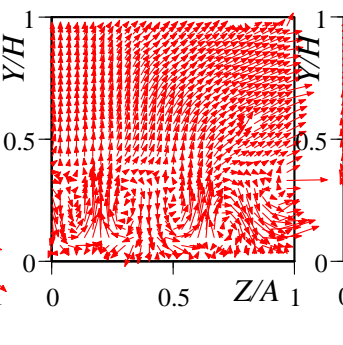

(b) $X / B=-1$

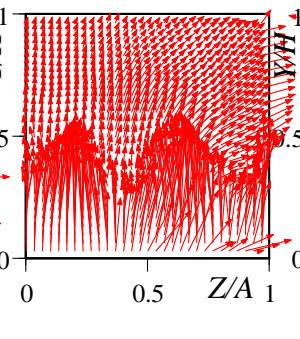

(c) $X / B=-0.5$

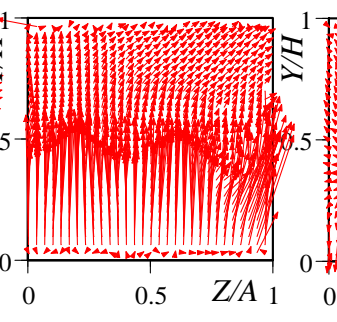

(d) $X / B=0$

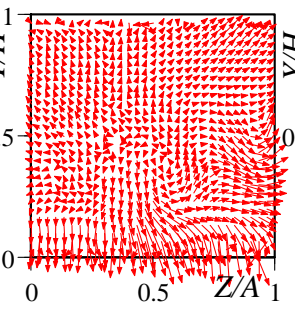

(e) $X / B=1$

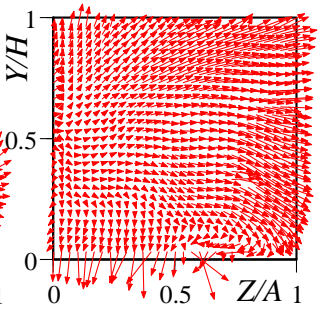

(f) $X / B=2$

Fig. 10 Streamwise evolution of the secondary flow mean velocity vectors $\left(\theta=30^{\circ}\right)$

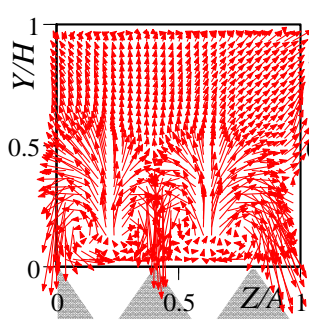

(a) $X / B=-2$

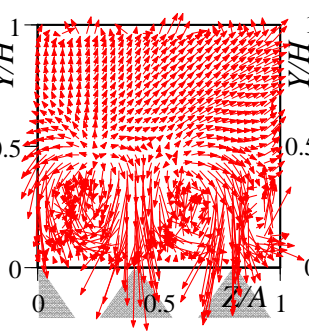

(b) $X / B=-1$

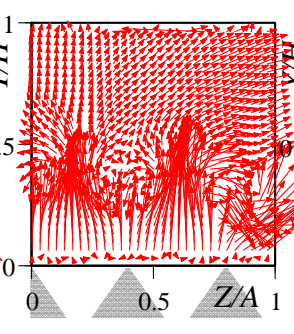

(c) $X / B=-0.5$

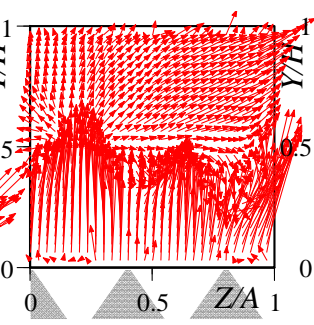

(d) $X / B=0$

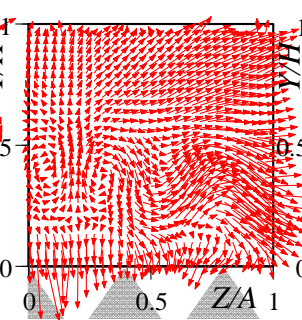

(e) $X / B=1$

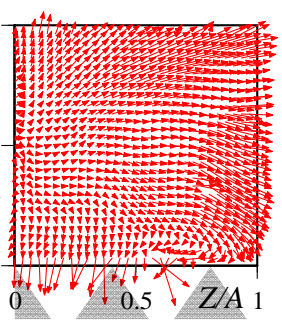

(f) $X / B=2$

Fig. 11 Streamwise evolution of the secondary flow mean velocity vectors $\left(\theta=60^{\circ}\right)$

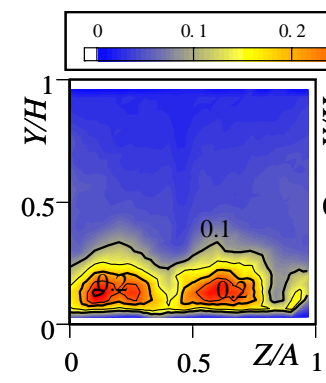

(a) $X / B=-2$

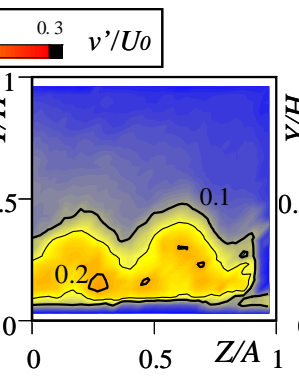

(b) $X / B=-1$

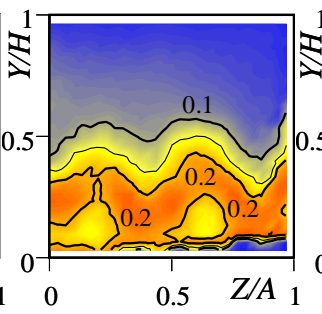

(c) $X / B=-0.5$

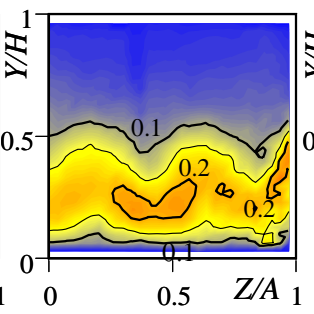

(d) $X / B=0$

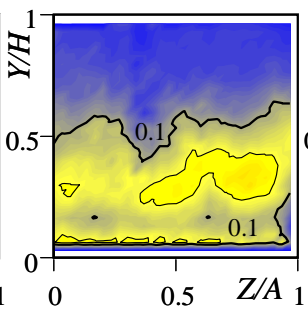

(e) $X / B=1$

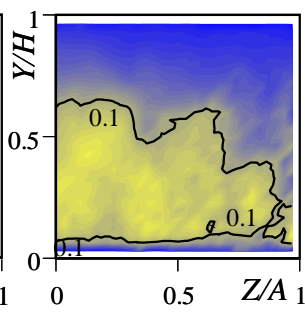

(f) $X / B=2$

Fig. 12 Streamwise variation of the turbulence intensity $v^{\prime} / U_{0}\left(\theta=30^{\circ}\right)$

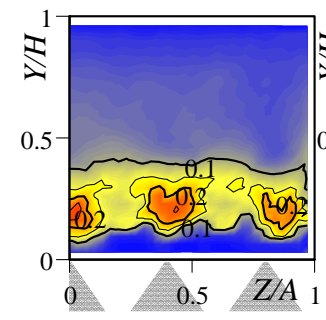

(a) $X / B=-2$

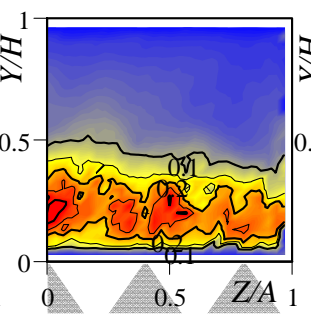

(b) $X / B=-1$

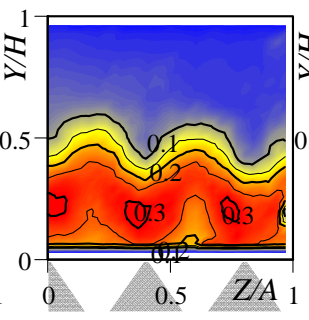

(c) $X / B=-0.5$

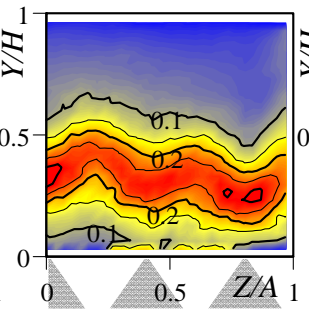

(d) $X / B=0$

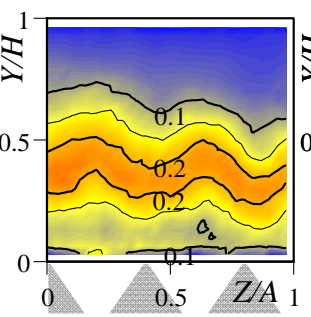

(e) $X / B=1$

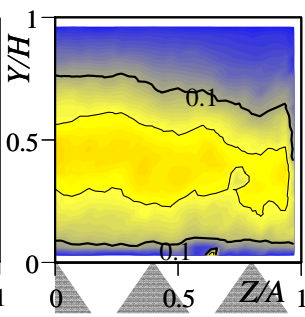

(f) $X / B=2$

Fig. 13 Streamwise variation of the turbulence intensity $v^{\prime} / U_{0}\left(\theta=60^{\circ}\right)$

gradient $\partial \mathrm{V} / \partial \mathrm{Z}$ observed in Fig. 10, and it follows that the interaction between the longitudinal vortices generated in the main flow and the branch flow that enters the main channel produces high turbulence in the flow merging region. Then $v^{\prime}$ decreases gradually in the streamwise direction, but $v^{\prime}$-values inside the mixing layer is much larger than those observed in the standard channel (Fig. 6) and the turbulent mixing of hot and cold airflows is promoted effectively.

Figure 13 shows the results for $\theta=60^{\circ}$. At $X / B=-2$, the local maximums of $v^{\prime} / U_{0}$ are observed not between the wings but on the extensions of the wing bisectors. In the cross sections of $X / B>-0.5, v^{\prime}$ distributions for $\theta=60^{\circ}$ are qualitatively similar to those for $\theta=30^{\circ}$ but the values of $v^{\prime} / U_{0}$ are generally larger than those for $\theta=30^{\circ}$. Figure 14 compares spanwise distributions of $v^{\prime}$ measured at $X / B=2$ on the line of $Y / H=0.5$ in the standard channel and the channel with $\theta=30^{\circ}$ and $\theta=60^{\circ}$. It is again ascertained that the distribution of $v^{\prime}$ for $\theta=60^{\circ}$ is qualitatively similar to that for $\theta=30^{\circ}$, but the values are about $30 \%$ larger. This increase of $v^{\prime}$ contributes to further promotion of turbulent mixing with larger angle of attack of wings.

Figure 15 shows a comparison of $\left(T-T_{c}\right) /\left(T_{h}-T_{c}\right)$ and $v^{\prime} / U_{0}$ measured at $X / B=2$ and $Z / A=0.4$ in the standard channel and the channel with the delta-wing row of $\theta=60^{\circ}$. $v^{\prime}$ in the channel with the wings is much increased in the region corresponding to the upper part of the thermal mixing 


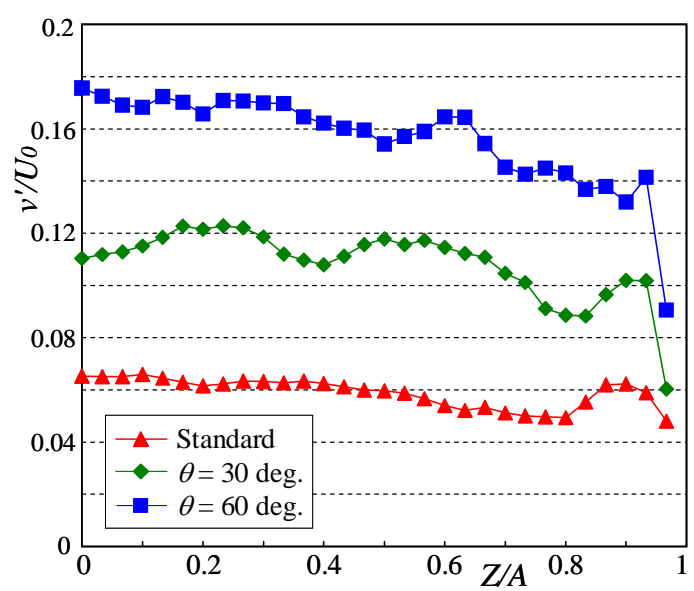

Fig. 14 Comparison of spanwise distributions of $v^{\prime}$

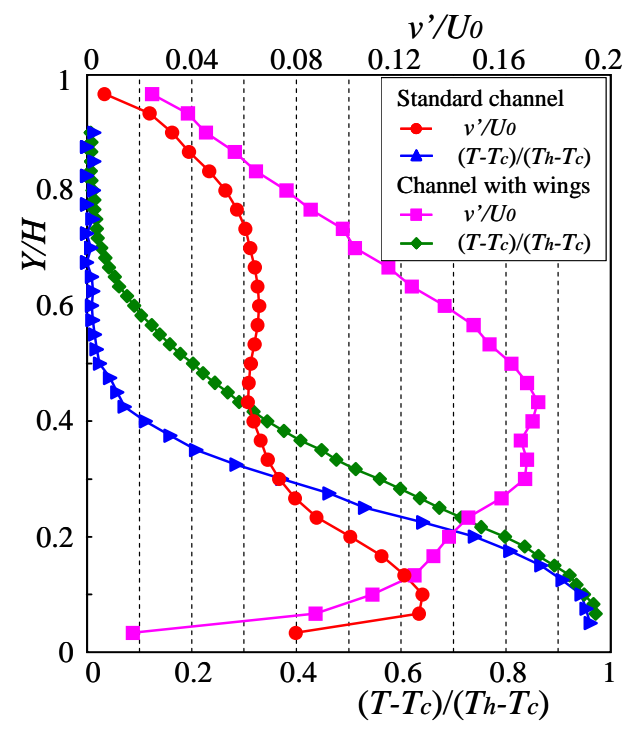

Fig. 15 Comparison of $\left(T-T_{c}\right) /\left(T_{h}-T_{c}\right)$ and $v^{\prime} / U_{0}$ at $Z / A=0.4$

layer in the standard channel. It is understood that this increase of $v^{\prime}$ contributes to the promotion of turbulent heat transport in the $Y$-direction, i.e., the upward development of the thermal mixing layer.

\section{CONCLUSIONS}

The results of this study are summarized as follows.

(1) The turbulent mixing of hot and cold airflows is promoted effectively by the delta-wing row settled at the bottom wall of the main channel upstream the flow-merging region. At small angle of attack of the wings $(\theta)$, the mean temperature shows an uneven distribution in the spanwise direction. As $\theta$ is increased, the thermal mixing layer becomes thicker and the spanwise unevenness of the temperature distribution is gradually relieved.

(2) The mixedness is defined to evaluate quantitatively the degree of mixing of the hot and cold airflows and soundness of the mean temperature distribution in the mixing layer. The mixedness increases almost linearly with respect to $\theta$. This demonstrates that we can control the turbulent thermal mixing of hot and cold airflows by changing the angle of attack of the delta wings.

(3) A pair of longitudinal vortices with a common-flow down is produced just after each delta wing, but they break by the merging of two flows. High turbulence is produced just downstream the delta wings. The component in the vertical direction $v^{\prime}$ once decrease in the main-flow direction but increases to attain the maximum in the flow-merging region. Large $v^{\prime}$ exists inside the thermal mixing layer and can promote the turbulent mixing of hot and cold airflows. The values of $v^{\prime}$ in the mixing layer increases as $\theta$ is increased.

\section{REFERENCES}

[1] C. Bruecker. Study of the Three-Dimensional Flow in a T-junction Using a Dual-Scanning Method for Three-Dimensional

Scanning-Particle-Image-Velocimetry (3-D SPIV). Exp. Thermal Fluid Science, 14: 35-44, 1997.

[2] H. L. Wu, X. F. Peng and T. K. Chen. Influence of Sleeve Tube on the Flow and Heat Transfer Behavior at a T-junction. Int. J. Heat Mass Transfer, 46: 2637-2644, 2003.

[3] J.-M. Ndombo and R. J.A. Howard. Large Eddy Simulation and the effect of the turbulent inlet conditions in the mixing Tee. Nuclear Eng. Design, 241: 2172-2183, 2011.

[4] M. Kitada, H. Asano, M. Kanbara and S. Akaike. Development of Automotive Air-Conditioning System Basic Performance Simulator: CFD Technique Development, JSAE Review, 21: 91-96, 2000.

[5] M. Hirota, E. Mohri, H. Asano and H. Goto. Experimental Study on Turbulent Mixing Process in Cross-flow type T-junction. Int. J. Heat Fluid Flow, 31: 776-784, 2010.

[6] J.-P. Bonnet, W.L. Siauw, S. Bourgois and J. Tensi. Influence of a synthetic jet excitation on the development of a turbulent mixing layer. Int. J. Heat Fluid Flow, 29: 957-966, 2008.

[7] M. Hirota, M. Kuroki, H. Nakayama, H. Asano and S. Hirayama. Promotion of Turbulent Thermal Mixing of Hot and Cold Airflows in T-junction, Flow Turbulence Combust., 81: 321-336, 2008.

[8] M. C. Gentry and A. M. Jacobi. Heat Transfer Enhancement by Delta-Wing-Generated Tip Vortices in Flat-Plate and Developing Channel Flows. J. Heat Transfer, 124: 1158-1168, 2002.

[9] T.-M. Liou, C.-C. Chen and T.-W. Tsai. Heat Transfer and Fluid Flow in a Square Duct With 12 Different Shaped Vortex Generators. J. Heat Transfer, 122: 327-335, 2000.

[10]D. You, M. Wang, R. Mittal and P. Moin. Large-Eddy Simulations of Longitudinal Vortices Embedded in a Turbulent Boundary Layer. AIAA Journal, 44: 3032-3039, 2006. 